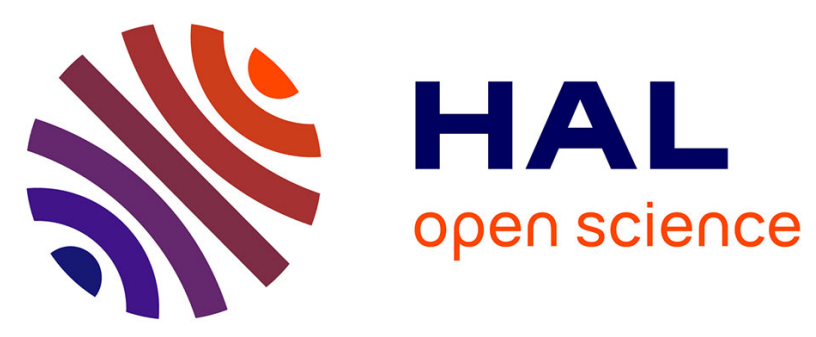

\title{
Residual stress fields analysis in rolled Zircaloy-4 plates: Grazing incidence diffraction and elastoplastic self-consistent model
}

\author{
David Gloaguen, Jamal Fajoui, Baptiste Girault
}

\section{- To cite this version:}

David Gloaguen, Jamal Fajoui, Baptiste Girault. Residual stress fields analysis in rolled Zircaloy-4 plates: Grazing incidence diffraction and elastoplastic self-consistent model. Acta Materialia, 2014, 71, pp.136 - 144. 10.1016/j.actamat.2014.02.031 . hal-01007254

\section{HAL Id: hal-01007254 \\ https://hal.science/hal-01007254}

Submitted on 22 Jan 2017

HAL is a multi-disciplinary open access archive for the deposit and dissemination of scientific research documents, whether they are published or not. The documents may come from teaching and research institutions in France or abroad, or from public or private research centers.
L'archive ouverte pluridisciplinaire HAL, est destinée au dépôt et à la diffusion de documents scientifiques de niveau recherche, publiés ou non, émanant des établissements d'enseignement et de recherche français ou étrangers, des laboratoires publics ou privés. 


\title{
Residual stress fields analysis in rolled Zircaloy-4 plates: Grazing incidence diffraction and elastoplastic self-consistent model
}

\author{
D. Gloaguen, J. Fajoui, B. Girault \\ GeM, Institut de Recherche en Génie Civil et Mécanique (UMR CNRS 6183), Université de Nantes-Centrale Nantes, IUT de Saint-Nazaire, 58 rue Michel \\ Ange, BP 420, 44606 Saint-Nazaire Cedex, France
}

\begin{abstract}
An experimental and theoretical investigation was carried out to study the in-depth distribution of residual stress after a cold-rolling test in a zirconium alloy. Grazing incidence X-ray diffraction was used to analyze the heterogeneous stress field with regard to different diffraction volumes below the surface of the sample. An interpretation of the intergranular stress analysis based on in-depth stress development has been made using an elastoplastic self-consistent model in order to account for the effect of plastic anisotropy. The contribution and the magnitude of both the first- and second-order residual stresses as a function of the penetration depth has been correctly evaluated using information from the model. The results show the complementarity of the methods used.
\end{abstract}

Keywords: Zirconium; Plasticity; X-ray diffraction; EPSC model; Grazing incident method

\section{Introduction}

The fabrication of metallic components with appropriate structural properties has always been a real challenge for thermomechanical processing. Owing to their hexagonal close-packed (hcp) structure, $\mathrm{Zr}$ alloys exhibit highly anisotropic plastic properties at both mesoscopic (grain) and macroscopic levels with various active deformation modes. These properties and the crystallographic texture explain the appearance and development of important residual stresses during mechanical treatments. These stresses are termed intergranular or second-order stresses. They depend on initial and induced crystallographic textures. The engineering consequences of second-order stresses, in particular with strong preferential crystallographic orientation, can be severe: texture and stresses at a given step in the fabrication process will affect the formability in which the next mechanical process might be realized.

Diffraction experiments provide information about the mechanical behavior of polycrystalline grains groups in the near-surface volume during thermomechanical treatment. Using X-ray diffraction (XRD) as an analytical tool, the change in the lattice parameters due to strain occurrence is measured through the induced diffraction peak shift in order to determine the residual stresses [1-3]. Further to a thermomechanical process, residual stresses can be generated by inhomogeneous plastic deformation upon two length scales, one given by the size of the metallic piece and the other by the size of the grains forming the polycrystalline aggregate. The inhomogeneity on the sample length scale gives the macroscopic stress field or first-order stresses (denoted $\sigma^{\mathrm{I}}$ ), while inhomogeneity on the grain size scale gives the intergranular or second-order stresses (denoted $\left.\sigma^{\mathrm{II}}\right)$. The two are superimposed, and XRD measurements give a combination of first- and second-order stresses $[3,4]$. In order to determine first-order residual stresses, 
intergranular stresses must thus be subtracted from the measured stress field. The fundamental question is thus how to obtain the macroscopic strain from the measured strain in a hexagonal structure, observed with the help of a specific $\{$ hk.l\} plane, when we have a superposition of both macroscopic and important intergranular contributions.

The case of rolling process is more complicated since it leads to the development of in-depth residual stress (first- and second-order) inhomogeneity. In this case, the well-established standard methods of X-ray residual stress analysis, such as the $\sin ^{2} \psi$ method, are not suitable since X-ray penetration depth varies significantly during the measurement series (in most cases, between 1 and $100 \mu \mathrm{m}$ thick) and no homogeneous macroresidual stress state can be assumed in the surface layer sampled by the X-ray beam.

Over the past three decades, numerous X-ray measuring techniques have been developed, focusing especially on residual stress field analysis in the near-surface zone of polycrystalline bulk materials and in thin films. A description of the different diffraction methods for stress gradients analysis is given in Refs. [5-7]. For an investigation of depth-dependent stress distribution in the near-surface, the grazing incidence X-ray diffraction (GIXD) method has proved very popular for a wide range of crystalline materials [8-10]. The depth from which diffraction information is collected can be controlled by selecting a suitable incident beam angle (with respect to sample surface) or wavelength. GIXD enables the stress gradients to be determined from diffraction measurements at different effective penetration depths by varying the angle of incidence.

Whatever the method used, a macroscopic residual stress gradient is assumed in most cases. This assumption is, however, no longer valid for plastically deformed polycrystalline materials and especially for hexagonal aggregates such as $\mathrm{Zr}$ alloys. After a mechanical loading, such as that used in metal-forming processes, plastic anisotropy induces large plastic incompatibility stresses [11]. These intergranular plastic stresses must be taken into account for a proper interpretation of X-ray data.

In this work, we propose a new development of the stress analysis method based on GIXD in order to correctly analyze the first- and second-order in-depth stress inhomogeneity of a hexagonal material. More generally, the purpose of this study is to combine experimental observations (XRD) with the predictions of an elastoplastic self-consistent (EPSC) model in order to obtain more information about the different factors responsible for the appearance of residual second-order stresses. This comparison allows a better understanding and interpretation of diffraction and mechanical results.

In return, the capacity to measure intergranular strains provides an experimental tool to understand how intergranular strains are generated. Diffraction data offers a rigorous test of the models at a microscopic level $[12,13]$. Comparison with experimental residual stresses should allow a more accurate interpretation of scale transition approach such as the EPSC model. In fact, plastic deformation of $\mathrm{Zr}$ alloys is accommodated by a complex mixture of crystallographic slip and deformation twinning. It is difficult to identify the role played by the different deformation mechanisms on the overall behavior. It is still unclear which deformation systems are actually activated in a polycrystal during straining.

\section{Grazing-incidence X-ray diffraction}

\subsection{Diffraction geometry}

The GIXD method is based on a modification of conventional Bragg-Brentano geometry to provide an asymmetric diffraction result which allows access to small depths in the sample by varying the incident angle. The incident angle, $\alpha$ (i.e. the angle between the incident $\mathrm{X}$-ray beam and sample surface), is adjusted by changing the $\omega$ diffractometer tilt and is fixed during measurement. The detector is moved along the goniometer circle ( $2 \theta$ angle) and records the diffraction profile of several reflecting $\{$ hk.1\} lattice planes. For this geometry, the angle $\psi$ between the measuring direction $\mathrm{Q}$ and the normal of the sample surface depends on the incidence angle $\alpha$ and lattice planes $\{$ hk.1\} (Fig. 1):

$\psi=\theta-\alpha$.

The tilt angle is then specific to each diffraction peak: the angles $\psi$ and $\theta$ cannot be chosen independently for a given angle $\alpha$. Therefore, in a single $2 \theta$ scan, a range of $\psi$ angles is automatically selected when a number of Bragg peaks with different Miller indices are measured at different $2 \theta$ angles (Fig. 1).
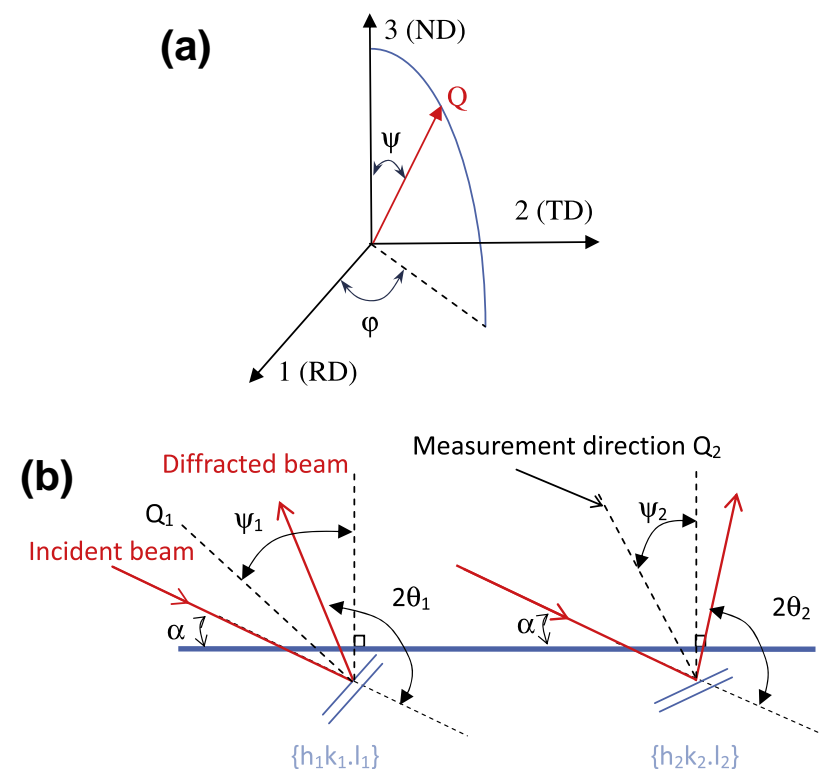

Fig. 1. (a) Orientation of the measurement direction with respect to the specimen system S. RD, TD and ND stand for rolling, transverse and normal direction, respectively. (b) Schematic diagram of the GIXD geometry illustrating the various angular relations. 
In a GIXD experiment, the $\alpha$ angle is the key parameter since it controls the penetration depth of the X-ray in the material. It is conveniently described by the $1 /$ e penetration depth $\tau$ :

$\tau=\frac{1}{\mu} \frac{\sin \alpha \sin (2 \theta-\alpha)}{\sin \alpha+\sin (2 \theta-\alpha)}$,

where $\mu$ is the linear absorption coefficient for a given material and wavelength.

In Fig. 2a, the penetration depth is displayed as a function of $\sin ^{2} \psi$ for $\mathrm{Cu} K_{\alpha}$ and $\mathrm{Cr} K_{\alpha}$ radiation in the studied Zy-4 alloy and according to Eq. (2). As shown in Fig. 2a, for a given $\alpha$ angle, GIXD provides an almost constant penetration depth over a wide range of $\psi$ angles, contrary to conventional diffraction method. Stress can be analyzed for different layer thicknesses under the sample surface by chosing different incident angle values. When the incident angle is relatively low (typically $<5^{\circ}$ ), as is the case for GIXD, the refraction which shifts the diffraction peaks towards higher $2 \theta$ angles should be considered. That is why the diffraction peaks have been corrected for X-ray refraction [14]. Corrections have been also made for other factors which can be significant for the final result in the GIXD method, i.e. Lorentz-polarization and absorption factors [3].
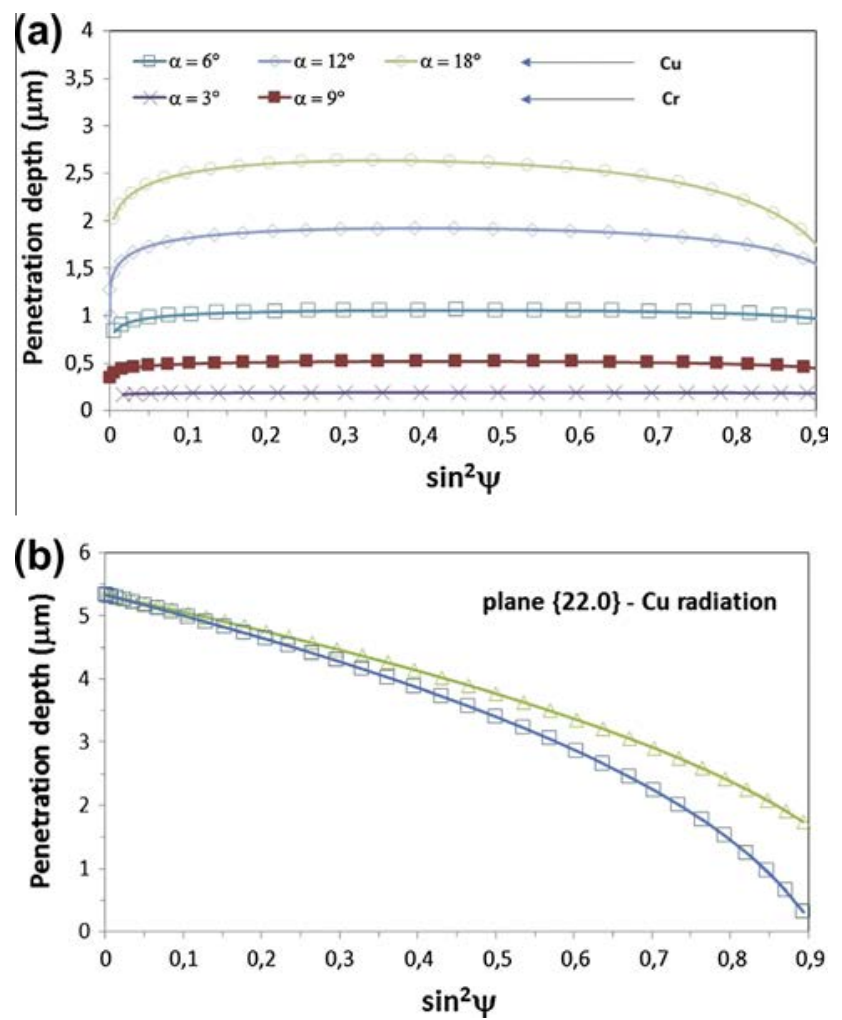

Fig. 2. Variation of the penetration depth vs. $\sin ^{2} \psi$ in Zy-4: (a) GIXD method (different incidence angles, $\alpha$ ) with $\mathrm{Cu} K \alpha$ and $\mathrm{Cr} K \alpha$ radiation (employing Eq. (2)); (b) conventional diffraction stress analysis for the $\{22.0\}$ reflection with $\mathrm{Cu} K \alpha$ radiation ( $\square$ : $\omega$ mode, $\Delta: \chi$ mode).

\subsection{Residual stress analysis}

We present succinctly the principles of residual stress determination by XRD and the key role played by elastic and plastic anisotropy properties on data interpretation. More details can be found in Refs. $[1-4,15]$. In the present work, the method proposed by Baczmanski et al. for cubic material [15] has been used and extended to hexagonal material [11] in the context of GIXD geometry.

As a first step, it may be helpful to summarize the diffraction stress analysis. The elastic strain $\langle\varepsilon(h k . l, \varphi, \psi)\rangle_{V_{d}}$ of a grain group having common $\{$ hk.l\} plane-normals, parallel to the diffraction vector Q (characterized by azimuthal and inclination angles, respectively $\varphi$ and $\psi$, as introduced in Fig. 1), i.e. grains fulfilling diffraction conditions, can be calculated from the measured lattice spacing $\langle d(h k . l, \varphi, \psi)\rangle_{V_{d}}$ and a reference one $d_{0}(h k . l)$ using the following expression:

$\langle\varepsilon(h k . l, \varphi, \psi)\rangle_{V_{d}}=\frac{\langle d(h k . l, \varphi, \psi)\rangle_{V_{d}}-d_{0}(h k . l)}{d_{0}(h k . l)}$,

where $d_{0}(h k . l)$ is the strain-free lattice parameter of the $\left\{\right.$ hk.1\} planes, and \langle\rangle$_{\mathrm{Vd}}$ indicates an average over diffracting grains for the considered $\{$ hk. 1$\}$ reflection.

$\langle d(h k . l, \varphi, \varphi)\rangle_{V_{d}}$ is calculated using the well-known Bragg's law once the $2 \theta$ angle has been determined from the measured diffraction peak. The strain in the $\mathrm{Q}$ direction is then given by:

$\langle\varepsilon(h k . l, \varphi, \psi)\rangle_{V_{d}}=\ln \left(\frac{\sin \theta_{0}(h k . l)}{\sin \theta(h k . l, \varphi, \psi)}\right)$,

where $\theta_{0}$ is the Bragg angle of the stress-free material.

In order to determine the macroscopic stress tensor $\sigma^{\mathrm{I}}$, such measurements have to be performed along different suitably chosen directions (defined by $\psi$ and $\varphi$ angles). $\langle\varepsilon(h k . l, \varphi, \psi)\rangle_{V_{d}}$ values for those directions can be employed to derive the residual stress tensor:

$\langle\varepsilon(h k . l, \varphi, \psi)\rangle_{V_{d}}=F_{i j}(h k . l, \varphi, \psi) \sigma_{i j}^{I}$,

where $F_{i j}(h k . l, \varphi, \psi)$ are the diffraction stress factors for the $\{$ hk.1\} reflection [5]. These factors can be calculated from single-crystal elastic data of the grains composing the aggregate and the crystallographic orientation distribution function (ODF) after adopting a suitable grain-interaction model. It should be noted that this relation remains valid for macroscopically elastically anisotropic specimens.

If significant intergranular strains are present at the mesoscopic scale in the material after a thermomechanical stimulus, the stress tensor $\sigma_{i j}^{I}$ determined from relation (5) can depend on the $\{$ hk.1\} family analyzed, i.e. the measured strain cannot be identified as the macroscopic strain if the material presents anisotropic properties. This behavior has been already observed in a previous work [11] on cylindrical Zircaloy-4 samples in a cold-worked state. Strain measurements in the tangential and longitudinal directions have been carried out on $\{10.4\}$ and $\{30.2\}$ 
diffracting planes. Axial stress analysis has shown an opposite sign for the two different diffracting planes: $+189 \pm 16$ and $-376 \pm 14 \mathrm{MPa}$ for the $\{10.4\}$ and $\{30.2\}$ planes, respectively.

This measured stress is related to the sum of strain incompatibilities at the macroscopic and mesoscopic levels. As a consequence of the single-crystal anisotropic properties, the response of the grains within the aggregate is different from that of isolated crystals. This is responsible for the development of internal stresses. Plastic intergranular strains (and stresses) are induced during plastic deformation because a full accommodation of each grain shape is prevented by the neighboring grains since the plastic strain varies from one crystallite to another.

Consequently, the measured strain is composed of two terms, and Eq. (5) can be rewritten as:

$$
\begin{aligned}
\langle\varepsilon(h k . l, \varphi, \psi)\rangle_{V_{d}}= & F_{i j}(h k . l, \varphi, \psi) \sigma_{i j}^{I} \\
& +\left\langle\varepsilon^{\text {IIIlasticity }}(h k . l, \varphi, \psi)\right\rangle_{V_{d}} .
\end{aligned}
$$

More details about the establishment of this relation can be found in Refs. $[15,16]$.

$\left\langle\varepsilon^{\text {IIplasticity }}(h k . l, \varphi, \psi)\right\rangle_{V_{d}}$ is the plastic intergranular strain, averaged over the volume of diffracting grains, for a given $\{$ hk.1\} reflection and a particular scattering vector orientation. It represents the elastic strain caused by the plastic strain misfit in grains in relation to the surrounding matrix and contributing to the measured diffraction peak position shift.

Eq. (6) clearly shows that the measured strain cannot be identified with the macroscopic strain when the material presents plastic anisotropic properties. The presence of intergranular strain after a mechanical solicitation influences the measured strain. Generally, the interpretation of experimental data is based on the unjustified assumption that $\left\langle\varepsilon^{\text {IIplasticity }}(h k . l,, \varphi, \psi \tau)\right\rangle_{V_{d}}=0$. In our study, we propose to quantify the importance of these intergranular stresses and show how they affect the interpretation of the experimental results.

Based on the methodology proposed by Baczmanski et al., some information obtained from the scale transition method has been used. The mesoscopic elastic strain tensor resulting from the rolling process can be predicted by the EPSC model for each grain. The average strain for diffracting grain volume, $\left\langle\varepsilon^{\text {IIth }}(h k . l, \varphi, \psi)\right\rangle_{V_{d}}$ (where the superscript "th" means theoretical model), can thus be calculated. As shown in Ref. [15], the predicted $\left\langle\varepsilon^{I t h}(h k . l, \varphi, \psi)\right\rangle_{V_{d}}$ depends on the hardening and relaxation processes which cannot be accurately accounted for in the models. Only the variation (and not the magnitude) of residual strains originating from plasticity (in relation to $\varphi$ and $\psi$ ) can be correctly predicted. Backmanski et al. [15] have proposed the introduction of an unknown scaling factor $q$ in order to determine the real amplitude of elastic strains (or stresses) of plastic origin.

Hence, the second term in Eq. (6) is assumed to be approximated by: $\left\langle\varepsilon^{\text {IIlasticity }}(h k . l, \varphi, \psi, \tau)\right\rangle_{V_{d}}=q\left\langle\varepsilon^{\text {Ilth }}(h k . l, \varphi, \psi)\right\rangle_{V_{d}}$.

The case of a rolling process is more complicated since it results in an in-depth residual stress gradient. The measured lattice strain depends on the penetration depth $\tau$, and Eq. (6) should be rewritten as:

$$
\begin{aligned}
\langle\varepsilon(h k . l, \varphi, \psi \tau)\rangle_{V_{d}}= & F_{i j}(h k . l, \varphi, \psi) \sigma_{i j}^{I}(\tau) \\
& +\left\langle\varepsilon^{\text {IIlasticity }}(h k . l, \varphi, \psi \tau)\right\rangle_{V_{d}} .
\end{aligned}
$$

It is necessary at this stage to make an additional assumption to obtain a feasible method for stress gradient analysis:

$\left\langle\varepsilon^{\text {IIplasticity }}(h k . l, \varphi, \psi \tau)\right\rangle_{V_{d}}=q(\tau)\left\langle\varepsilon^{\text {IIth }}(h k . l, \varphi, \psi)\right\rangle_{V_{d}}$.

Using a scale transition model, we predict values of the residual stress and strain tensor for every crystalline orientation, and calculate the average strain $\left\langle\varepsilon^{\text {Ilth }}(h k . l, \varphi, \psi)\right\rangle_{V_{d}}$ for the diffracting volume knowing $F_{i j}(h k . l, \phi, \psi)$, the theoretically predicted strain $\left\langle\varepsilon^{I t h}(h k . l, \varphi, \psi)\right\rangle_{V_{d}}$ and the measured deformations $\langle\varepsilon(h k . l, \varphi, \psi \tau)\rangle_{V}$. The other unknown quantities from Eq. (9) $\left(q(\tau)\right.$ and $\left.\sigma_{i j}^{I}(\tau)\right)$ can be determined using a non-linear fitting procedure. This allows a complete description of the stress (and strain) field in a plastically deformed polycrystalline material for a given penetration depth. In this method, the intergranular strain gradient is taken into account through $q(\tau)$. For each $\tau$-value, the amplitude of $\left\langle\varepsilon^{I I t h}(h k . l, \varphi, \psi)\right\rangle_{V_{d}}$ is rescaled by varying the $q(\tau)$ value through the fitting procedure described above. Thus, an estimation of the intergranular stress (and strain) gradient can be obtained along with the real contribution of the first-order stress to measured lattice strains.

\section{Experiments}

\subsection{Samples}

In the present study, a cold-rolled Zircaloy-4 (Zy-4) sheet is considered. Its chemical composition in wt. $\%$ is: Sn (1.4), Fe (0.21), Cr (0.09), O (0.12), with balance $\mathrm{Zr}$. The aggregate exhibits equiaxed grains with a mean size of $25 \mu \mathrm{m}$. The specimen has been submitted to a coldrolling test at room temperature along the original rolling direction (RD) of the sheet. The total strains were equal to $30.5 \%$. To analyze the mechanical behavior of the material at large deformation, one sample (dimensions: $92 \mathrm{~mm} \times 16 \mathrm{~mm} \times 2 \mathrm{~mm}$ ) has been cut along the RD of the sheet.

\subsection{Texture analysis}

XRD analysis was performed with a four-circle XRD3003PTS Seifert goniometer using $\mathrm{Cu} K_{\alpha}$ radiation. The X-ray beam output collimator was $0.5 \mathrm{~mm}$ in diameter. The diffraction peaks were recorded with a positionsensitive detector. We measured incomplete pole figures $(\mathrm{PFs})$ on a $5^{\circ} \times 5^{\circ}$ grid with tilt and azimuth angles ranging 
from $0^{\circ}$ to $60^{\circ}$ and $0^{\circ}$ to $360^{\circ}$, respectively. For each experimental direction, the diffraction pattern (measured in sheet plane) has been fitted using a non-linear least-squares analysis and assuming pseudo-Voigt peak profiles in order to evaluate background noise and to obtain the intensities of the peaks. The ODF calculation was performed with experimental PFs $\{00.2\},\{10.1\},\{110\}$ and $\{10.3\}$ with the help of the WIMV algorithm implemented in the BEARTEX program package [17]. Initial textures are shown in Fig. 3a. The texture of the undeformed sample corresponds to recrystallization due to the thermal treatment following the sheet-forming process. The texture is characterized by a reinforcement of the $c$-axis around the normal direction (ND). The prismatic PF exhibits intensity maxima along the RD. Fig. $3 \mathrm{~b}$ also shows the PF after the mechanical test. A "classic" texture evolution is observed for prismatic and basal PFs [18]. In the final state, the $\{00.2\}$ PF shows a bimodal disposition of the intensity maxima in the NT-TD plane at about $21^{\circ}$ from the ND axis.

\subsection{Stress analysis}

GIXD measurements were carried out employing a three-circle $(2 \theta, \omega, \varphi)$ Seifert Calypso XRD diffractometer with a parallel beam geometry. The beam was focused onto

(a)
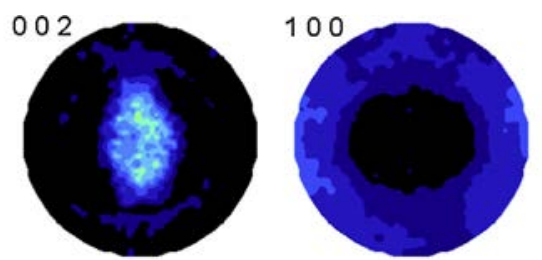

(b)
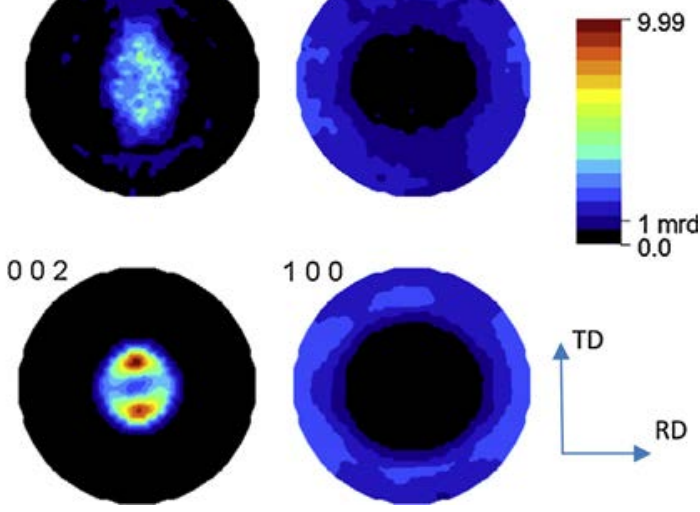

RD

Fig. 3. XRD pole figures obtained on $\{00.2\}$ and $\{10.0\}$ diffracting planes of rolled $\mathrm{Zy}-4$ for (a) $0 \%$ and (b) $30.5 \%$ strain (RD, rolling direction; $\mathrm{TD}$, transverse direction). the sample using a collimating polycapillary system allowing a divergence of $\sim 0.3^{\circ}$ on the sample surface. The incident beam diameter was $2 \mathrm{~mm}$.

Long Soller slits (with a divergence of $0.4^{\circ}$ ) were used to reduce the axial divergence (divergence in the plane perpendicular to the diffraction plan) of the diffracted X-ray beam detected by an energy-dispersive XRD detector (based on silicon drift detector technology). The measurements were made with $\mathrm{Cu} K_{\alpha}$ and $\mathrm{Cr} K_{\alpha}$ radiation.

The set of diffraction peaks obtained for all incidence angles has been fitted with a pseudo-Voigt function taking into account the $K_{\alpha 1}-K_{\alpha 2}$ contribution. The centroid of the fitted diffraction line was taken as the peak position. For each diffraction peak, background was fitted through a polynomial function. The measurement ranges were chosen in order to cover an adequate number of points for a complete description of peak tails and background. The counting times and step size were adjusted for each diffraction peak to obtain a sufficient peak-to-background ratio for accurate peak position determination. The diffraction stress factors were theoretically calculated with an elastic selfconsistent model [3]. The influence of the texture on these constants is taken into account by weighting single-crystal elastic constants with the texture function, i.e. ODF (2000 grains). In this study, we assume a biaxial stress state in the measured region: $\sigma_{\mathrm{i} 3}=0, \mathrm{i}=1,2,3$. The other parameters used for measurements are summarized in Table 1.

As a first step, the GIXD experimental setup was tested using various calibration powders of different materials, namely $\mathrm{Si}$ and $\mathrm{Fe}$ residual stress-free reference powders. For incidence angles, $\alpha$, ranging from $3^{\circ}$ to $15^{\circ},\langle\varepsilon(h k . l, \varphi, \psi \tau)\rangle_{V_{d}}$ were measured for the azimuths $\varphi=0^{\circ}$ and $90^{\circ}$ and plotted as a function of $\sin ^{2} \psi$ (Fig. 2a). The stress values were calculated from the relation (8), neglecting the second-order stresses. For these powders, no stress should be obtained irrespective of the $\alpha$ value. In other words, the measured lattice parameters $\langle a(h k . l, \varphi, \psi \tau)\rangle_{V_{d}}$ (deduced from Bragg's law and the spacing formula for cubic material) should be constant for the different chosen penetration depths. In Fig. 4, the measured $\langle a(h k . l, \varphi, \psi \tau)\rangle_{V_{d}}$ are displayed as a function of $\tau$ for the two powders. The plot of the lattice parameter leads to a straight horizontal line: the measured lattice parameter values do not depend on the depth. The mean values obtained for the different radiations and depths are: $\mathrm{a}_{\mathrm{Fe}}=2.8660 \pm 0.0002 \AA$ and $\mathrm{a}_{\mathrm{Si}}=5.4306 \pm 0.0001 \AA$.

Table 1

Parameters used for measurements and residual stress evaluations

\begin{tabular}{lllllll}
\hline & Radiation & $\alpha\left(^{\circ}\right)$ & $\tau$ range $(\mu \mathrm{m})$ & $2 \theta$ range $\left(^{\circ}\right)$ & Number of $\{$ hk.1\} reflections & $\mu\left(\mathrm{cm}^{-1}\right)$ \\
\hline Fe powder & $\mathrm{Cu}$ & $3,9,12,15$ & {$[0.20-0.83]$} & {$[79-141]$} & 5 & 2396 \\
& $\mathrm{Cr}$ & $3,6,9,12,15$ & {$[0.55-2.21]$} & {$[64-162]$} & 3 & 890 \\
Si powder & $\mathrm{Cu}$ & $3,4,5,8,10,12$ & {$[3.2-10.9]$} & {$[71-164]$} & 8 & 152 \\
& $\mathrm{Cr}$ & $3,5,6,9$ & {$[1-2.8]$} & {$[68-138]$} & 4 & 472 \\
Zy-4 plate & $\mathrm{Cu}$ & $3,6,9,12,18$ & {$[0.5-2.5]$} & {$[60-150]$} & 18 & 891 \\
& $\mathrm{Cr}$ & $3,6,9$ & {$[0.2-0.9]$} & {$[74-165]$} & 9 & 2595 \\
\hline
\end{tabular}




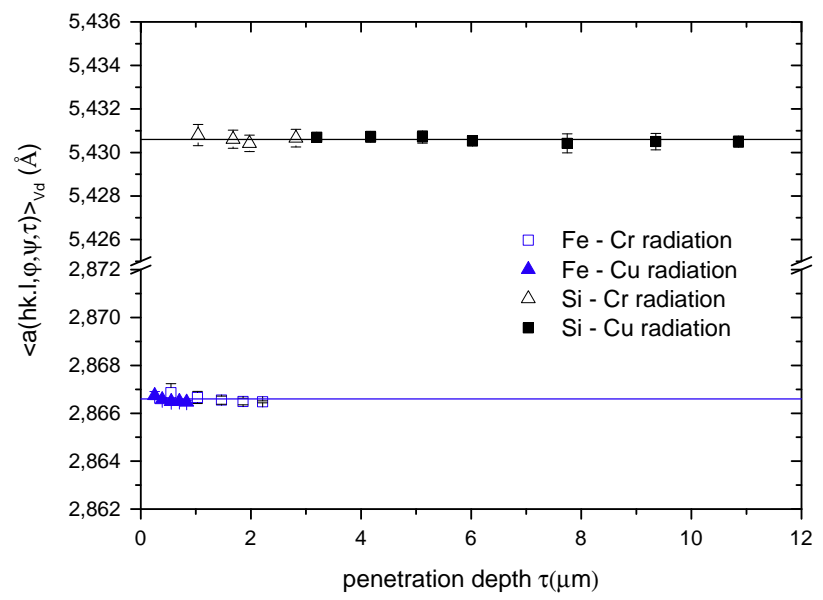

Fig. 4. Measured lattice constants $\psi$ vs. penetration depth $\tau$ for $\mathrm{Fe}$ and $\mathrm{Si}$ powders.

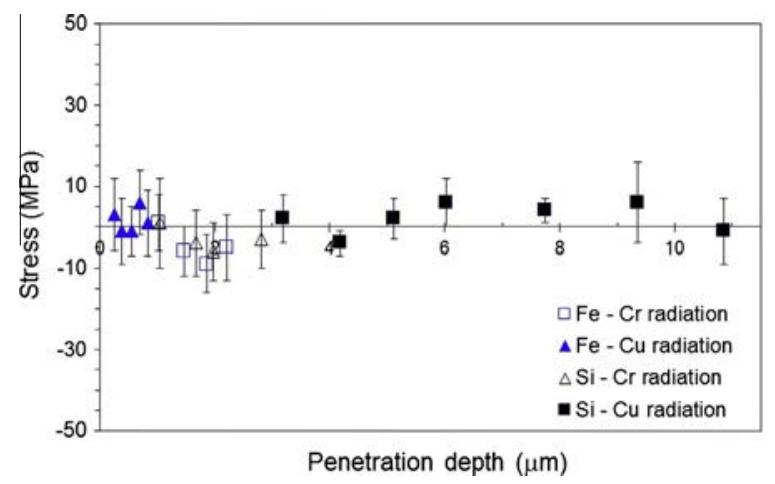

Fig. 5. Experimental stress component $\sigma_{11}$ plotted as a function of the penetration depth $\tau$.

The results of the of the $\sigma_{11}$ stress component determination are shown in Fig. 5. The residual stress values on the powder samples are low: $\sigma_{11}$ component varying from $-9 \pm 7$ up to $+6 \pm 8 \mathrm{MPa}$ for $\mathrm{Fe}$ and from $-4 \pm 5$ up to $+6 \pm 10 \mathrm{MPa}$ for Si. Irrespective of the penetration depth and radiation, this theoretical stress is lower than $10 \mathrm{MPa}$. This value corresponds to a systematic error and ultimately represents the precision of the method within the experimental setup used in this work.

\section{Elastoplastic polycrystal modelling}

\subsection{Model}

The principles for using the self-consistent model to predict elastoplastic deformation were proposed by Kröner and Hill $[19,20]$. In the present work, the model developed in Ref. [21] is used and will not be reviewed here. Refs. $[22,23]$ present a more detailed description of the EPSC model. Each grain is assumed to have the form of an ellipsoidal inclusion in a homogeneous effective medium, the properties of which are the average of all the other grains in the assembly. The measured initial texture was used as the input to the EPSC model: the polycrystal is represented by a weighted discrete distribution (2000 grains) of orientations (Euler angles $\left.\left(\varphi_{1}, \phi, \varphi_{2}\right)\right)$. Lattice rotations (reorientation by slip and twinning) and texture changes are included in the model [21]. The single-crystal elastic coefficients, crystallographic slip planes and directions are assigned to each crystallite and are used to calculate the shear of the grain under load. Hardening is accounted for in each deformation mode, g, by the use of a linear law: $\dot{\tau}_{c}^{g}=\sum_{r} H^{g r} \dot{\gamma}^{r}$, where $\dot{\tau}_{c}^{g}$ is the critical resolved shear stress (CRSS) rate, and $H^{g r}$ is the hardness of the matrix. Due to lack of relevant data on the nature of latent hardening in $\mathrm{Zr}$ alloys, the interactions between different deformation modes are described by a very simple law. The latent hardening coefficient is equal to the self-hardening coefficient: $H^{g r}=H^{g g}=H^{g}$.

\subsection{Data used in the simulations}

The single-crystal elastic constants used in this model are: $c_{11}=143.5 \mathrm{GPa}, \mathrm{c}_{12}=72.5 \mathrm{GPa}, \mathrm{c}_{13}=65.4 \mathrm{GPa}$, $\mathrm{c}_{33}=164.9 \mathrm{GPa}, \mathrm{c}_{44}=32.1 \mathrm{GPa}$ and $\mathrm{c}_{66}=35.5 \mathrm{GPa}$ [24]. The initial CRSSs are taken as identical for all systems of a given type.

Unlike cubic alloys, hexagonal materials, such as $\mathrm{Zr}$ alloys (with a c/a ratio $<1.633$ ), are characterized by a large variety of possible deformation systems: prismatic glide $\{10 \overline{1} 0\} 11 \overline{2} 0$ is reported to be the main active deformation mode [18,25]. Pyramidal $\langle\mathrm{c}+\mathrm{a}\rangle \operatorname{slip}\{10 \overline{1} 1\} 11 \overline{2} 3$, basal slip $\{0002\} 11 \overline{2} 0$ and pyramidal $\langle a\rangle\{10 \overline{1} 1\} 11 \overline{2} 0$ slip are generally presented as secondary slip modes [18,26], more or less activated depending on grain size, crystal orientation and material composition. At room temperature, twinning has also been observed in $\mathrm{Zr}$ samples [27,28]. Twinning of $\{10 \overline{1} 2\}$ and $\{11 \overline{2} 1\}$ types is expected in tension along the $\langle c\rangle$ axis, whereas twinning of $\{11 \overline{2} 2\}$ and $\{10 \overline{1} 1\}$ types is expected in compression along the $\langle\mathrm{c}\rangle$ axis. Plastic deformation of $\mathrm{Zr}$ alloys is accommodated by a complex mixture of crystallographic slip and deformation twinning. Based on a number of experimental observations, the deformation systems introduced in the model are assumed to be: prismatic slip denoted $\operatorname{pr}\langle\mathrm{a}\rangle$, pyramidal slip (pyr $\langle\mathrm{c}+\mathrm{a}\rangle$ and pyr $\langle\mathrm{a}\rangle)$, basal slip (bas $\langle\mathrm{a}\rangle)$ and $\{10 \overline{1} 2\}$ twinning (ttw). In the first step, the effect of deformation modes on the predicted strain (and stress) and texture was systematically analyzed. Each deformation mode was applied independently within the EPSC model, in order to observe its effect on strain accumulation and texture. The effect of combining two or three systems was also examined. The values and signs of the predicted strains differed much more than those detected experimentally. The observed trends and magnitudes of the texture and strains were not reproduced correctly by the model in the case of one or two deformation modes. Finally, all five deformation systems were applied simultaneously with different sets of material parameters (CRSS, matrix hardness). Basal slip was not included in the set of deformation modes because 
Table 2

Plastic parameters used in modelling.

\begin{tabular}{llll}
\hline$\tau^{p r\langle a\rangle}(\mathrm{MPa})$ & $\tau^{p y r\langle a\rangle}(\mathrm{MPa})$ & $\tau^{p y r\langle c+a\rangle}(\mathrm{MPa})$ & $\tau^{t t w}(\mathrm{MPa})$ \\
90 & 125 & 290 & 220 \\
$H^{p r\langle a\rangle}(\mathrm{MPa})$ & $H^{p y r\langle a\rangle}(\mathrm{MPa})$ & $H^{p y r\langle c+a\rangle}(\mathrm{MPa})$ & $H^{t t w}(\mathrm{MPa})$ \\
80 & 140 & 220 & 230 \\
\hline
\end{tabular}

some discrepancies appear with the X-ray results if this slip system is an active deformation mode. The best agreement was found using the values given in Table 2. In this study, we have used a single set of material parameters to describe the entire data set (the crystallographic texture and the residual elastic strains measured for the 18 different grains families).

\section{Results and discussion}

In a first step, stress analysis has been performed using the usual $\sin ^{2} \psi$ method, thus neglecting both residual stress gradient in the near-surface and intergranular stresses:

$\langle\varepsilon(h k . l, \varphi, \psi)\rangle_{V_{d}}=F_{i j}(h k . l, \varphi, \psi) \sigma_{i j}(h k . l)$

Strain measurements have been performed on three plane families: $\{10.4\} \quad\left(\varphi=0^{\circ}\right.$ and $\left.90^{\circ}\right),\{22.0\} \quad\left(\varphi=0^{\circ}\right.$ and $\left.90^{\circ}\right),\{00.4\}\left(\varphi=90^{\circ}\right)$ and $\{20.2\}\left(\varphi=0^{\circ}\right)$. For the $\{00.4\}$ and $\{20.2\}$ planes, measurements have not been carried out along $\varphi=0^{\circ}$ due to the texture.

At $\varphi=0^{\circ}$ (i.e. along the RD), the two planes $\{104\}$ and $\{20.2\}$ exhibit a similar compressive behavior with a stress value varying from one to another. The longitudinal stress value reaches $-224 \pm 14 \mathrm{MPa}$ for $\{10.4\},-258 \pm 30 \mathrm{MPa}$ for $\{22.0\}$ and $-350 \pm 12 \mathrm{MPa}$ for $\{20.2\}$.

$\mathrm{X}$-ray measurements show the effective existence of plastic anisotropy. As seen in Eq. (6), the measured stresses depend on the plane family analyzed. Strain incompatibilities are present at the mesoscopic level in the material, and consequently, the stresses obtained by XRD depend on the plane. The diffracting crystals are not the same for each case, allowing us to deduce that different second-order stresses exist, related to a strong anisotropic plastic deformation for these two plane families. The same behavior is observed with the stress values at $\varphi=90^{\circ}$ for the $\{10.4\}$, $\{22.0\}$ and $\{00.4\}$ planes: $\sigma_{22}(h k . l)$ value reaches $-173 \pm 10 \mathrm{MPa}$ for $\{10.4\},-300 \pm 20 \mathrm{MPa}$ for $\{22.0\}$ and $-25 \pm 10 \mathrm{MPa}$ for $\{00.4\}$.

The EPSC model has been used to simulate the residual intergranular strains and crystallographic texture after the rolling process. The mesoscopic stresses were then averaged and projected on the $\varphi \psi$ directions in order to simulate the diffracting volume behavior and crystallographic reorientation.

We now present a comparison between the experimental and calculated textures obtained with the EPSC formulation for the $30.5 \%$ cold-rolled samples. Fig. 6 shows the basal and prismatic pole figures, respectively, predicted by the EPSC calculations. (a)
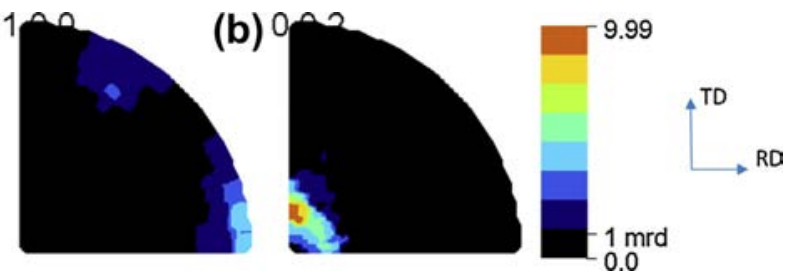

Fig. 6. Calculated prismatic (a) and basal (b) pole figures representing the deformation textures of the rolled $\mathrm{Zy}-4$.

As shown in Fig. 6, there is good agreement between the experimental results and the EPSC model. The principal features of the experimental texture are reproduced by the simulations. We are able to clearly predict the basal poles preferentially oriented at $21^{\circ}$ from RD in the RD-TD plane. With the CRSS values chosen above, deformation is accommodated through $\operatorname{pr}\langle a\rangle$ and $\operatorname{pyr}\langle\mathrm{c}+\mathrm{a}\rangle$; the relative contributions of pyr $\langle\mathrm{a}\rangle$ and ttw are small. At the end of the loading, the proportions of activated systems are: $52.5 \%$ for prismatic mode, $34.4 \%$ for pyramidal slips $\langle\mathrm{c}+\mathrm{a}\rangle, 13.1 \%$ for $\operatorname{pyr}\langle\mathrm{a}\rangle$ and $0 \%$ for tensile twinning. Finally, we obtain a reasonable distribution, in agreement with the experimental observations: prismatic glide is the main active mechanism and pyramidal slip is the secondary deformation mode, while twinning contributes very little to plastic deformation. The weak activation of the tensile twinning is normal because it reorientates preferentially the grains which have their $\langle c\rangle$ axis close to the RD.

The theoretical values of the $\left\langle\varepsilon^{\text {Ilth }}(h k . l, \varphi, \psi)\right\rangle_{V_{d}}$ strain were predicted by the EPSC model for $30.5 \%$ strain. Applying Eq. (8) and fitting the results obtained from the model to the experimental data, the first-order stress tensor elements $\sigma_{11}^{I}(\tau)$ (along the $\mathrm{RD}$ ), $\sigma_{22}^{I}(\tau)$ (along the $\mathrm{TD}$ ) and the $\mathrm{q}(\tau)$ factor have been determined. This procedure has been applied for different penetration depths $\tau$ to determine the first-order stress gradient with the two radiations. In this case, the $\mathrm{q}(\tau)$ factor takes into account the intergranular strain variations with depth.

To obtain quantitative information on the in-depth stress distribution, we have applied the formalism given in Section 2.2 to our data. The results of the $\sigma_{11}^{I}(\tau)$ and $\sigma_{22}^{I}(\tau)$ stress component determination according to Eqs. (8) and (9) are summarized in Table 3. The data are given as a function of the penetration depth (Eq. (2)). The sample shows a compressive stress along those two directions that decreases with depth and which was introduced by plastic deformation of the sample surface during the rolling process. In the transverse direction, the observed values are smaller than the corresponding stress data for $\sigma_{11}^{I}(\tau)$.

In order to visualize the results of the calculation and the fitting procedure quality, the $\langle\varepsilon(h k . l, \varphi, \psi, \tau)\rangle_{V_{d}}$ strains (evaluated according to Eq. (8)) and those measured by XRD have been plotted as a function of $\sin ^{2} \psi$ in Fig. 7 and compared; for the sake of clarity, only two cases are shown. The predicted results agree with the fact that, in the $\langle\varepsilon(h k . l, \varphi, \psi, \tau)\rangle_{V_{d}}$ vs. $\sin ^{2} \psi$ representation of the 
Table 3

In-depth distribution of residual stress components for the Zy-4 sample determined by GIXD and sin ${ }^{2} \psi$ methods.

\begin{tabular}{|c|c|c|c|c|c|}
\hline Penetration depth $(\mu \mathrm{m})$ & $\alpha\left(^{\circ}\right)$ & $\sigma_{11}^{I}(\mathrm{MPa})$ & & $\sigma_{22}^{I}(\mathrm{MPa})$ & \\
\hline 0.19 & $3(\mathrm{Cr})$ & $-306 \pm 27$ & & $-165 \pm 25$ & \\
\hline 1.04 & $6(\mathrm{Cu})$ & $-289 \pm 25$ & & $-150 \pm 37$ & \\
\hline 1.88 & $12(\mathrm{Cu})$ & $-271 \pm 35$ & & $-135 \pm 29$ & \\
\hline 2.51 & $18(\mathrm{Cu})$ & $-260 \pm 33$ & & $-120 \pm 31$ & \\
\hline \multirow{3}{*}{\multicolumn{2}{|c|}{ Stress analysis with the standard $\sin ^{2} \psi$ method }} & $\{10.4\}$ & $\{20.2\}$ & $\{10.4\}$ & $\{00.4\}$ \\
\hline & & $\varphi=0^{\circ}$ & $\varphi=0^{\circ}$ & $\varphi=90^{\circ}$ & $\varphi=90^{\circ}$ \\
\hline & & $-224 \pm 14$ & $-350 \pm 12$ & $-173 \pm 10$ & $-25 \pm 10$ \\
\hline
\end{tabular}
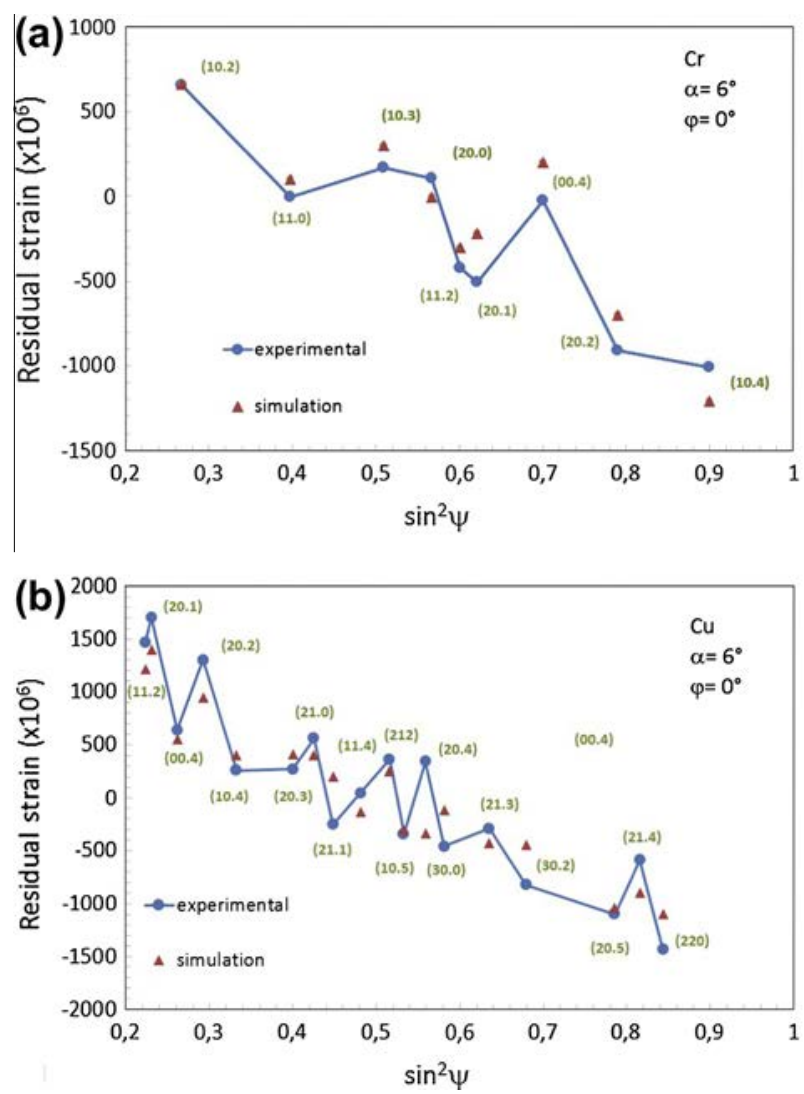

Fig. 7. Measured diffraction strain (filled circles) and theoretical results (filled triangles) estimated from Eq. (8) for the different studied reflections of the rolled Zy-4 sample as a function of $\sin ^{2} \psi\left(\varphi=0^{\circ}\right)$. Two cases are shown: (a) $\alpha=6^{\circ}$ with $\mathrm{Cr}$ radiation and (b) $\alpha=6^{\circ}$ with $\mathrm{Cu}$ radiation.

deformed samples, the best fitting for the 18 studied planes is obtained with prismatic slip as the main deformation mode.

In order to show the influence of intergranular strain introduced by plastic deformation, the in-depth distribution of first-order stress have been calculated without the $\left\langle\varepsilon^{\text {IIIlasticity }}(h k . l, \varphi, \psi, \tau)\right\rangle_{V_{d}}$ term: $\langle\varepsilon(h k . l, \varphi, \psi, \tau)\rangle_{V_{d}}=$ $F_{i j}(h k . l, \varphi, \psi) \sigma_{i j}^{I}(\tau)$. In this case, at $\tau=1.04 \mu \mathrm{m}\left(\alpha=6^{\circ}\right.$ with $\mathrm{Cu}$ radiation), $\sigma_{11}^{I}(\tau)$ and $\sigma_{22}^{I}(\tau)$ are equal, respectively, to $-113 \pm 41$ and $-142 \pm 40 \mathrm{MPa}$. This example allows us clearly to conclude that the $\left\langle\varepsilon^{\text {IIplasticity }}(h k . l, \varphi, \psi, \tau)\right\rangle_{V_{d}}$ term plays a crucial role in the proper interpretation of the XRD results.
XRD probes coherently diffracting domains (CDDs) that are smaller than or equal to the grain size. Consequently, although the X-ray penetration depth $(2.5 \mu \mathrm{m})$ covers only a tenth of the grain size $(25 \mu \mathrm{m})$, a certain number of CDDs remain irradiated due to the spot size $\left(>20 \mathrm{~mm}^{2}\right)$. Therefore, even though these CDDs are scarce, they provide a reasonable description of the material behavior that is consistent with the numerical approach [29]. Even though a good correlation is observed between the numerical and experimental results, certain deficiencies still remain. The influence of a free surface is not considered here, whereas owing to absorption of X-rays, surface grains contribute more to diffraction than the grains that lie deeper in the sample. The anisotropic interactions between grains in the near-surface volume on the calculated diffraction stress factors would need to be considered in the future [30] to improve the agreement between experimental and theoretical results.

A comparison with experimental texture and residual strains constitutes an accurate and relevant validation of the choice of deformation systems. It is a good way to evaluate the overall agreement between model and experiment. For example, it is possible to explain and reproduce the texture without the pyr $\langle a\rangle$ system but the model can then no longer simulate correctly the experimental residual stresses for all $\varphi$ directions. These four systems with $\operatorname{pr}\langle\mathrm{a}\rangle$ as the main deformation mode are the only ones that can explain and reproduce each experimental result obtained by XRD.

\section{Conclusion}

A method for the determination of the first- and secondorder stress gradients in a plastically deformed polycrystalline hop alloy was proposed and tested. The stress gradient has been determined by performing lattice strain measurements at constant and adjustable penetration depths via the GIXD method. The stress state of a cold-rolled Zy-4 sample was investigated by employing the standard $\sin ^{2} \psi$ analysis as well as a new approach. It has been shown that the presence of a stress gradient in the surface-adjacent region of the investigated layer could only be determined quantitatively by measurements at fixed penetration depths. It has also been demonstrated that, using this method, residual macrostresses can be evaluated and separated from the plastic incompatibility stresses for textured samples, using 
some additional information from the EPSC model, i.e. intergranular strains induced by plastic deformation.

This constitutive model is applied to predict the crystallographic texture and residual intergranular strain based on a crystallographic treatment of twinning and slip within individual grains after a cold-rolling test. Using a single set of hardening parameters, the model notably achieved reasonable agreement in texture evolution and in-depth distribution of residual stress for all XRD analyses performed in this study, although it is not possible to consistently reproduce all the details of the residual lattice strain evolution. The present study highlights the usefulness of a polycrystal model to explore the active deformation modes in hcp alloys and shows the complementarity of the methods used. The classical $\sin ^{2} \psi$ method cannot be used for this purpose since penetration depth varies during the experiment and the influence of second-order strain must be taken into account to obtain a correct interpretation of the XRD results for hexagonal material.

\section{References}

[1] Dölle HJ. Appl Cryst 1979;12:489.

[2] Noyan IC, Cohen JB. Residual stress-measurements by diffraction and interpretation. Heidelberg: Springer Verlag; 1987.

[3] Hauk V. Structural and residual stress analysis by nondestructive methods. Amsterdam: Elsevier; 1997.

[4] Ortiz M, Pochettino AA. J Nucl Mater 1996;229:65.

[5] Welzel U, Ligot J, Lamparter P, Vermeulen AC, Mittemeijer EJ. J Appl Cryst 2005;38:1.

[6] Skrzypek J, Baczmanski A, Ratuszek W, Kusior E. J Appl Cryst 2001;34:427.

[7] Birkholz M. Thin film analysis by X-ray scattering. Weinheim: WileyVCH Verlag; 2006.

[8] Van Acker K, De Buyser L, Celis JP, Van Houtte P. J Appl Cryst 1994;27:56.
[9] Angerer P, Neubauer E, Yu LG, Khor KA. Int J Refract Met Hard Mater 2009;27:1004.

[10] Dudognon J, Vayer M, Pineau A, Erre R. Surf Coat Technol 2006;200:5058

[11] Gloaguen D, Berchi T, Girard E, Guillén R. J Nucl Mater 2008;374:138.

[12] Dakhlaoui R, Braham C, Baczmanski A, Wronski S, Wierzbanowski K, Olivier EC. Mater Sci Forum 2006;524:185.

[13] Gloaguen D, François M, Guillén R, Royer J. Phys Status Solidi (a) 2002;193:12.

[14] Wronski S, Wierzbanowski K, Baczmanski A, Braham C, Lodini A Mater Sci Forum 2008;571:289.

[15] Baczmanski A, Wierzbanowski K, Lipinski P, Helmholdt RB, Ekambaranathan G, Pathiraj B. Phil Mag A 1994;69:437.

[16] Baczmanski A, Hfaiedh N, François M, Wierzbanowski K. Mater Sci Eng A 2009;501:153.

[17] Wenk HR, Matthies S, Donavan J, Chateigner D. J Appl Cryst 1998;31:262.

[18] Tenckhoff E. Deformation mechanisms, texture and anisotropy in zirconium and zircaloy. Philadelphia: ASTM; 1988.

[19] Kröner E. Acta Metall 1961;9:155.

[20] Hill R. J Mech Phys Solids 1965;13:89.

[21] Gloaguen D, Oum G, Legrand V, Fajoui J, Branchu S. Acta Mater 2013;61:5779.

[22] Lipinski P, Berveiller M. Int J Plasticity 1989;5:149.

[23] Hutchinson W, Proc R. Soc London A 1970;319:247.

[24] Simmons G, Wang H. Single crystal elastic constants and calculated aggregate properties. Cambridge MA: MIT Press; 1971.

[25] Pochettino AA, Gannio N, Vial Edwards C, Penelle R. Scripta Metall 1992;27:1859.

[26] Akhtar A. Acta Met 1973;21:1.

[27] Akhtar A. Met Trans 1975;6A:1105.

[28] Akhtar A. J Nucl Mater 1973;47:79.

[29] Mabelly P, Hadmar P, Desvignes M, Sprauel JM. Assessment by micromechanical computation of the influence of internal stresses on the diffraction peak's broadening, Mechanics in Design, Sa. Meguid Ed., University of Toronto; 1996;1029.

[30] Wohlschlögel M, Baumann W, Welzel U, Mittemeijer EJ. Mater Sci Forum 2006;524:19. 